\title{
Cognitive Training Enhances Intrinsic Brain Connectivity in Childhood
}

\author{
Duncan E. Astle, ${ }^{1}$ Jessica J. Barnes, ${ }^{1}$ Kate Baker, ${ }^{2}$ Giles L. Colclough, ${ }^{3}$ and Mark W. Woolrich ${ }^{3}$ \\ ${ }^{1}$ Medical Research Council, Cognition and Brain Sciences Unit, and ${ }^{2}$ Department of Medical Genetics, University of Cambridge, Cambridge CB2 7EF, United \\ Kingdom, and ${ }^{3}$ Oxford Centre for Human Brain Activity, University of Oxford, Oxford OX3 7JX, United Kingdom
}

In human participants, the intensive practice of particular cognitive activities can induce sustained improvements in cognitive performance, which in some cases transfer to benefits on untrained activities. Despite the growing body of research examining the behavioral effects of cognitive training in children, no studies have explored directly the neural basis of these training effects in a systematic, controlled fashion. Therefore, the impact of training on brain neurophysiology in childhood, and the mechanisms by which benefits may be achieved, are unknown. Here, we apply new methods to examine dynamic neurophysiological connectivity in the context of a randomized trial of adaptive working memory training undertaken in children. After training, connectivity between frontoparietal networks and both lateral occipital complex and inferior temporal cortex was altered. Furthermore, improvements in working memory after training were associated with increased strength of neural connectivity at rest, with the magnitude of these specific neurophysiological changes being mirrored by individual gains in untrained working memory performance.

Key words: Cognitive training; development; electrophysiology; magnetoencephalography; working memory

\section{Introduction}

Working memory — the ability to hold in mind and manipulate small amounts of information for brief periods of time-is a key requirement for many everyday tasks and is considered a critical influence on educational progress during childhood (Gathercole et al., 2003). Training working memory can result in task performance benefits that transfer to similarly structured, but untrained tasks (Holmes et al., 2009; Dunning et al., 2013). Cognitive training has therefore been considered of potential therapeutic benefit for the remediation of cognitive deficits associated with neurodevelopmental disorders (Klingberg et al., 2005), acquired brain damage (Westerberg et al., 2007) and aging (Anguera et al., 2013). However, despite the growing interest in cognitive training and claims made about its potential, we know very little about the neural mechanisms by which training influences cognitive abilities. This is because it is difficult to combine longitudinal neuroimaging investigation with systematically

\footnotetext{
Received 0ct. 31, 2014; revised Feb. 2, 2015; accepted Feb. 21, 2015.

Author contributions: D.E.A. designed research; D.E.A. and J.J.B. performed research; D.E.A., J.J.B., G.L.C., and M.W.W. analyzed data; D.E.A., J.J.B., K.B., G.L.C., and M.W.W. wrote the paper.

D.E.A. was supported by a postdoctoral fellowship from the British Academy and intramural funding from the UK Medical Research Council (MRC) (Grant MC-A060-5PQ41). M.W.W. was supported by the Wellcome Trust, the National Institute for Health Research (NIHR), the Oxford Biomedical Research Centre based at Oxford University Hospitals Trust, Oxford University, and the MRC (UKMEG Partnership GrantMR/K005464/1). G.L.C. was supported by the Research Councils UK Digital Economy Programme (Grant EP/G036861/1). KB was supported by an NIHR Academic Clinical Lectureship. We thank Kia Nobre for her guidance on the data analysis and interpretation, Peter Watson for his advice on the statistics, and John Duncan and Joni Holmes for their comments on an earlier draft of this manuscript.

The authors declare no competing financial interests.

This article is freely available online through the J Neurosci Author Open Choice option.

Correspondence should be addressed to Dr. Duncan Astle, MRC Cognition and Brain Sciences Unit, 15 Chaucer Road, Cambridge CB2 7EF, UK. E-mail: duncan.astle@mrc-cbu.cam.ac.uk.

DOI:10.1523/JNEUROSCI.4517-14.2015

Copyright $\odot 2015$ the authors $\quad 0270-6474 / 15 / 356277-07 \$ 15.00 / 0$
}

controlled interventions, and this is even more true in target populations such as children (Jolles et al., 2012; Rueda et al., 2012). As a result, no study has yet explored whether and how cognitive training alters neurophysiology during childhood.

Tasks that tax working memory recruit areas in frontal and parietal cortex, overlapping with networks associated with attentional control (for review, see Duncan, 2010). A popular account linking frontoparietal activity to working memory performance is that activity in these domain-general networks codes information about fluctuating task goals. During control-demanding tasks, long-range functional connections between frontoparietal networks and other cortical areas integrate this higher-order information with ongoing processing in lower-level domain-specific systems (Stokes, 2011). The dynamic regulation of this functional connectivity provides a basis for integrating and optimizing lowerlevel sensory processes necessary in attention-demanding situations or when attempting to maintain multiple items in working memory (Gazzaley and Nobre, 2012). Similar functional interactions have been implicated in working memory performance during childhood (Astle et al., 2014; Barnes et al., 2015). However, the extent to which the neurophysiological mechanisms underlying efficient working memory performance can be modified by training is unknown.

In this study, we tested whether functional connectivity is altered in childhood by intensive working memory training and, if so, whether intrinsic changes in connectivity "at rest" are associated with any improvements in children's memory capacity. To test these hypotheses, 27 typically developing children, aged 8-11 years old, completed 20 sessions of computerized working memory training at home. Children were randomly assigned to an adaptive or placebo condition. Before and after training, all children underwent a 9 min resting state magnetoencephalography (MEG) scan and completed standardized assessments of short- 


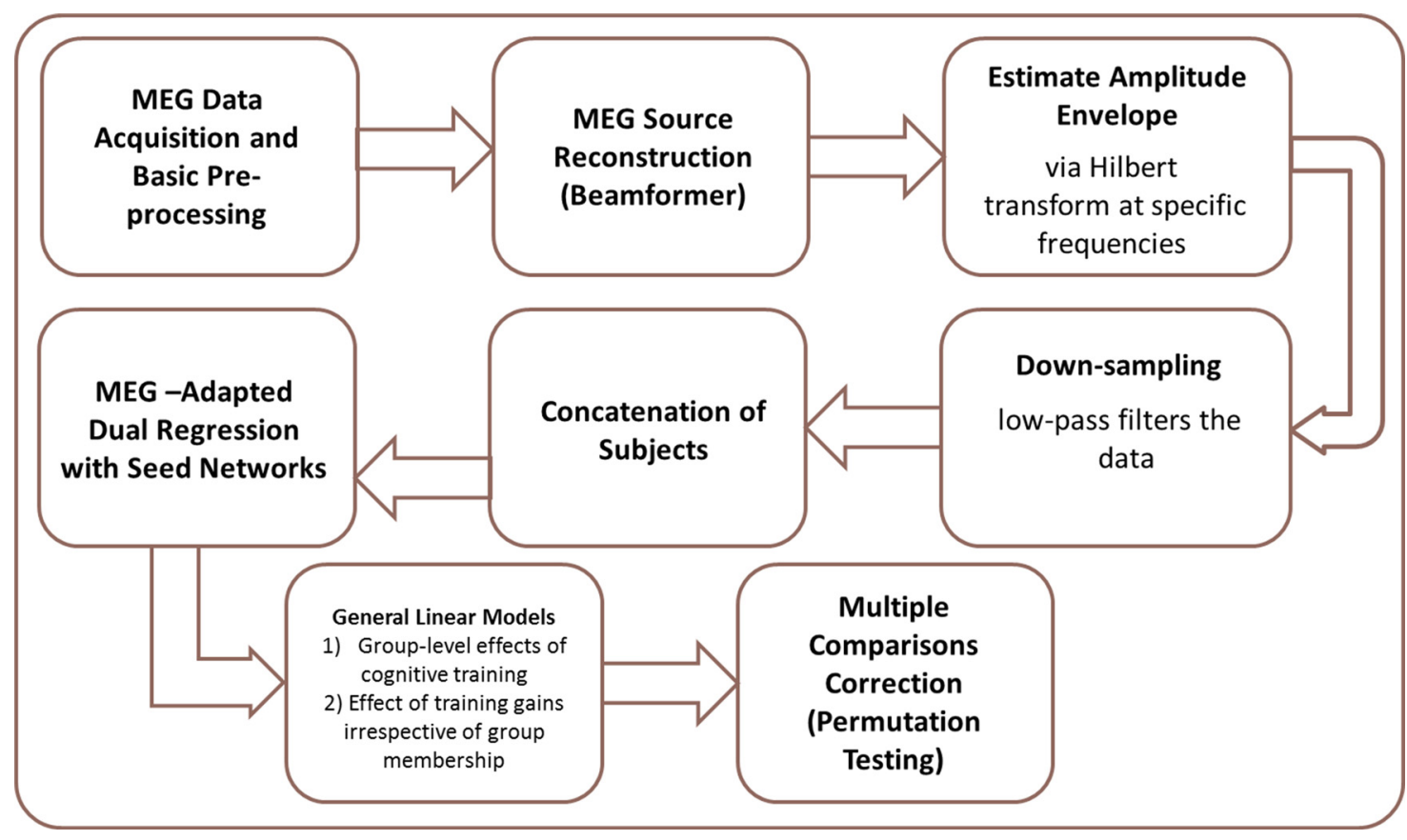

Figure 1. The MEG acquisition and analysis pipeline.

term and working memory. We then used resting-state MEG data to reconstruct electrophysiological oscillatory activity within the whole brain before and after training. Examining correlations in oscillatory activity allowed us to investigate whether cognitive training had any influence upon neurophysiological connections between frontoparietal networks and cortical areas typically recruited for working memory or attentional control and how these changes relate to gains in memory capacity measured outside the scanner.

\section{Materials and Methods}

Participants. A total of 33 children between 8 and 11 years of age $($ mean $=$ 119.2 months; $\mathrm{SD}=11.3$ months; 12 males) were recruited via local schools (see also Barnes et al., 2015). The only exclusion criterion was that children should not have a diagnosis of a developmental disorder or an acquired neurological condition. All participating children had normal or correctedto-normal vision. Children were randomly allocated to either the Adaptive or Placebo group. Six children failed to complete the training and did not return for the second session, so subsequent analysis was performed on the datasets from the remaining 27 children, 14 of whom had been allocated to the Placebo group and 13 to the Adaptive group. The Adaptive group included 3 boys with a mean age of 119.5 months ( $\mathrm{SD}=9.8$ months). The Placebo group included 7 males with a mean age of 118.8 months $(\mathrm{SD}=10.8$ months). There was no significant difference in age between the two groups $\left(t_{(25)}=0.182, p=0.857\right)$, the sex balance did not differ significantly between the groups $\left(x^{2}=2.095, p=0.148\right)$, and the two groups had equivalent IQ scores (as assessed using vocabulary and matrix reasoning scales from Wechsler Abbreviated Scale of Intelligence; $t_{(25)}=0.2265, p=0.8226$ ). Parents provided written informed consent and the study was approved by the University of Cambridge Psychology Research Ethics Committee.

Cognitive assessments. Before and after training we assessed each child's short-term and working memory ability using four assessments taken from the Automated Working Memory Assessment (AWMA; Alloway et al., 2008). We assessed each child's verbal short-term memory using a forward digit span procedure and their verbal working memory using a backward digit span task. We assessed each child's spatial short-term memory using a dot matrix task in which they had to retain a number of spatial locations and then report them in sequence. Each child's spatial WM was assessed using a spatial span task in which they had to retain the locations of a sequence of dots for subsequent recall while performing a series of mental rotations. To characterize our sample, we converted performance into age-independent standardized scores. For one child, we only had scores in the spatial domain. Across these assessments, there were no pretraining differences between the groups. Verbal short-term memory: Adaptive $=116(\mathrm{SD}=19)$ versus Placebo $=118(\mathrm{SD}=16$; $\left.t_{(24)}=0.397, p=0.695\right)$; verbal working memory: Adaptive $=106(\mathrm{SD}=$ $21)$ versus Placebo $=111\left(\mathrm{SD}=13 ; t_{(24)}=0.645, p=0.525\right)$; spatial short-term memory: Adaptive $=109(S D=15)$ versus Placebo $=107(S D=$ $\left.14 ; t_{(25)}=0.335, p=0.741\right)$; spatial working memory: Adaptive $=116$ $(\mathrm{SD}=14)$ versus Placebo $=115\left(\mathrm{SD}=11 ; t_{(25)}=0.139, p=0.891\right)$.

The initial short-term and working memory assessments were repeated after the respective training programs. Assessments at both time points were conducted by a member of the research team who was blinded to the group to which the children had been allocated. Parents were made aware of the existence of an intergroup experimental manipulation, but were not informed as to the nature of it. Children and parents were blinded to group allocation.

Cognitive training. Both groups performed the training at home and each child's progress was remotely monitored online by the research team. Children performed a minimum of 20 sessions, with an upper limit of 25 sessions, over 4-6 weeks. Each training session comprised 8 tasks (from a bank of 12 possible tasks). In each session, children performed 15 trials of each task, giving a total of 120 trials per training session (a total duration of 30-45 min per session). The children in the Adaptive group received a commercially available version of this program, which was not changed for these purposes, with task difficulty being adjusted on a trialby-trial basis according to the child's improving performance. Each task required children to retain either verbal or visual information for brief periods of time, often with concurrent processing or mental manipulation of the material required (see www.cogmed.com/rm for full details). The children assigned to the Placebo group completed an identical training program save for the fact that the tasks did not get progressively more difficult in response to the child's performance, with the span level set to two items across all trials (see also Dunning et al., 2013 and Holmes et al., 2009).

MEG data acquisition and basic preprocessing. Our MEG data acquisition and analysis pipeline can be seen in Figure 1; the details of these steps are provided in the following sections.

The same procedure for MEG data acquisition was used for both the pretraining and posttraining scans. MEG data were acquired with a highdensity whole-head VectorView MEG system (Elekta-Neuromag) containing a magnetometer and two orthogonal planar gradiometers at 102 
positions (306 sensors in total), housed in a magnetically shielded room. Data were sampled at $1 \mathrm{kHz}$ and signals slower than $0.01 \mathrm{~Hz}$ were not recorded. A 3D digitizer (FASTRACK; Polhemus) was used to record the positions of five head position indicator (HPI) coils and 50-100 additional points evenly distributed over the scalp, all relative to the nasion and left and right preauricular points. We also attached an electrode to each wrist to measure the pulse and bipolar electrodes to obtain horizontal (HEOGs) and vertical (VEOGs) electrooculograms. Head position was monitored throughout the recoding using the HPI coils. Particularly small children were seated on a booster seat to ensure that their head was optimally positioned within the scanner helmet. For resting-state data acquisition, children were instructed to close their eyes, let their mind wander, and not think of anything in particular for the duration of the scan. Data acquisition lasted $9 \mathrm{~min}$. All children were monitored by video camera throughout the scan and no child reported having fallen asleep.

External noise was removed from the MEG data using the signal-space separation method, and adjustments in head position within the recording were compensated for using the MaxMove software, both implemented in MaxFilter version 2.1 (Elekta Neuromag). At this stage, the data were downsampled to $250 \mathrm{~Hz}$. The continuous data were visually inspected and any short sections with large signal jumps were removed. A sensor-space temporal independent components analysis (ICA) was then used to remove artifacts arising from blinks, saccades, and pulse-related cardiac artifacts using a combination of metrics and manual inspection. The temporal ICA was conducted separately for each subject using fastICA run on the sensor space data and then the time course of each IC was correlated with the time course of the VEOG, HEOG, and cardiac channels, respectively. Components with a Pearson correlation value $>0.1$ with any of the artifact channels were subsequently removed from the data. Components dominated by $50 \mathrm{~Hz}$ noise were also removed.

MEG source reconstruction. For 23 of the children, we coregistered their MEG data to the child's T1-weighted structural MRI image acquired using a 3T Siemens Tim Trio and an MPRAGE sequence. For the remaining children, we used a standard MNI template (Barnes et al., 2015). For this coregistration, we used the digitized scalp locations and fiducials via an iterative closest point algorithm using SPM8 (http://www.fil. ion.ucl.ac.uk/spm/). beamforming the data were band-pass filtered to focus only on the slower frequencies (theta: $4-7 \mathrm{~Hz}$; alpha: $8-12 \mathrm{~Hz}$; lower beta: $13-20 \mathrm{~Hz}$; upper beta: $21-30 \mathrm{~Hz}$ ); previous work has shown that these slower frequencies are better for exploring functional connections with MEG and increase discrimination between spurious and genuine connectivity (Luckhoo et al., 2012). For each subject, source space activity was estimated at every vertex of a $6 \mathrm{~mm}$ grid covering the entire brain using a linearly constrained minimum variance beamformer (Van Veen et al., 1997). The beamformer combined information from both the magnetometers and planar gradiometers while taking into account the reduced dimensionality of the data introduced by the signal-space separation algorithm (Woolrich et al., 2011). Beamforming constructs a set of spatial filters that are applied to the sensor data to reconstruct the signal at each grid point with the aim of achieving unit band-pass response at the grid point while minimizing the variance passed from all other locations. This facilitates the identification of genuine functional connections rather than the effects of signal spread (Schoffelen and Gross, 2009) and acts to reduce noise. The process was repeated across all grid locations to achieve a whole-brain source reconstruction.

Hilbert envelope estimation, temporal down-sampling, and concatenation. Once the data were in source space, the oscillatory amplitude envelopes of the reconstructed time series were estimated via computation of the absolute value of the analytic signal, which was found using a Hilbert transform. This yielded an estimate of instantaneous signal amplitude at each voxel. The envelope time series for every voxel was low-pass filtered by dividing each envelope time course into $1 \mathrm{~s}$ windows and averaging within those windows to focus on low-frequency power fluctuations that are thought to be direct manifestations of electrophysiological functional connectivity (Brookes et al., 2011). Both weight-normalized and nonweight-normalized envelopes were estimated for use in the group-level (general linear model, GLM) analysis (Luckhoo et al., 2014). Spatial smoothing was also applied to the down-sampled envelope estimates (full-width half-maximum $=5 \mathrm{~mm}$ ). Finally, the children's data were temporally concatenated to produce a single dataset.
MEG-adapted dual regression. The next step of our analysis used the temporally concatenated down-sampled envelope data to explore functional connectivity. We used an independent canonical set of resting state networks (RSNs) taken from a recent functional connectivity (fc)-fMRI study (Smith et al., 2012) as a basis for the analysis and looked for changes specifically within the left and right lateral frontoparietal networks and bilateral frontoparietal networks. We chose these networks a priori because they represent our best approximation of those cortical networks particularly linked to attentional control and working memory in adulthood. We tested whether differences in these networks, or in the areas that communicate with them, might underpin any significant changes in working memory capacity stemming from the intervention. A further advantage of choosing networks a priori from this independent dataset is that the nodes are particularly focal, making it easier to unambiguously identify distinct frontoparietal networks and relate any findings across different studies (Barnes et al., 2015).

To extract subject specific maps for each of the fc-fMRI networks, we performed a MEG-adapted dual regression (DRMEG) analysis analogous to that used in fMRI data (Filippini et al., 2009). In the first stage of DRMEG, we performed a spatial regression of the fc-fMRI network maps on the concatenated weights-normalized envelopes to yield concatenated network time courses. In the second stage, we broke these concatenated time courses into subject specific blocks. For each subject, we performed a temporal regression of the network time course segment from the non-weight-normalized down-sampled envelopes. This gave a spatial map for each RSN that was specific to each subject but critically had an unbiased estimate of the true variance of activity for that RSN, which was essential for all subsequent multisubject statistics (Luckhoo et al., 2014). The result of the dual regression was that, for each child, we obtained a wholebrain map that corresponded to the voxel-by-voxel electrophysiological connectivity with each of these candidate networks before and after training. In short, this step enabled us to look at covariation between oscillatory activity in our networks of interest and the rest of the brain. Importantly, taking this whole-brain connectivity approach rather than simply looking at overall changes in the activity levels of particular RSNs enabled us to specify which, if any, neurophysiological connections correspond to training gains.

GLM. We then tested the hypothesis that specific connections with our candidate networks are altered by the intervention, using a whole-brain GLM. First, we tested for an interaction between time and group by including this as a regressor in our GLM. The result was a voxelwise GLM in which we could identify any significant interaction between time and group. We also controlled for any impact of initial ability on functional connectivity. We included variables identifying each subject as covariates, which effectively regresses out the subjects' means, leaving a paired test on within-subject effects. We also controlled for any impact of initial ability on functional connectivity.

However, this group-level approach is a relatively superficial way of testing the hypothesis that changes in working memory capacity and changes in intrinsic brain connectivity are significantly related. Simply because a child is assigned to the adaptive training program does not guarantee that there will be any change to their working memory capacity. Likewise, performing the placebo training each day may produce some benefits for some children. A more direct way of exploring the relationship between changes in brain connectivity and working memory capacity would be to conduct a GLM that incorporates information about the impact of the intervention across children regardless of group membership. Therefore, we complemented our first GLM with a subsequent analysis in which the first regressor corresponded to the relationship between working memory scores (taken as the mean raw score on the untrained assessments) and connectivity after training. The second regressor corresponded to the relationship between working memory scores and connectivity before training. In this second GLM, we could then explore the relationship between functional connectivity and working memory capacity after training while controlling for any relationship before training. This is analogous to testing for a relationship between changes in brain connectivity and changes in working memory capacity. As before, we also included regressors that corresponded to the subject mean for each child.

Correcting for multiple comparisons. The whole-brain outputs of our two GLMs were fed into a permutation procedure to correct for multiple 


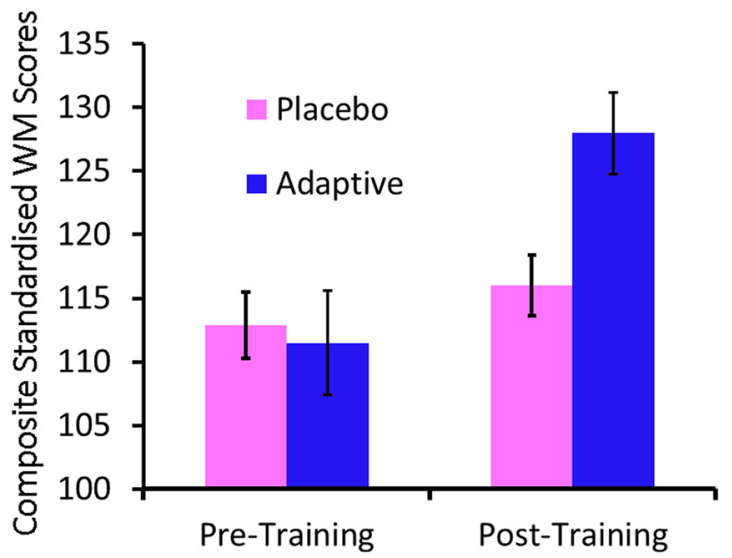

Figure 2. Performance on the standardized working memory assessments in both groups, before and after training. Error bars indicate the SEM.

comparisons. We adopted a standard procedure for testing the hypothesis that there are no contiguous regions where there are explained differences in resting-state connectivity. We did not use the standard clustering threshold of $p<0.05$ because, for each voxel, the effect of a particular GLM was repeated over three candidate networks and four frequencies. The threshold was Bonferonni corrected, which produced a clustering threshold of $p<0.004$. In practice, because two-tailed testing was conducted (because it is theoretically possible that training might be associated with either positive or negative effects), this meant thresholding at an upper and lower limit of alpha equal to 0.002 . Once we had used this threshold to identify any clusters of voxels that exceed this level and established the relative size of these clusters, we conducted a sign-flipping permutation procedure to produce a null distribution using 5000 permutations. We were then able to compare the size of each result to this null distribution, thereby identifying the relative alpha level and producing a $p$-value that controls for the multiple comparisons across the 8471 voxels. This nonparametric permutation approach has a number of advantages relative to more traditional approaches to significance testing with electrophysiological data: First, it makes no a priori assumptions about when or where effects are likely to be apparent; second, this approach accounts for multiple comparisons over space and time, restoring control to the false-positive rate (Kilner, 2013).

\section{Results}

\section{Adaptive training boosts working memory capacity}

After training, the Adaptive group demonstrated significant improvements in standardized scores on the untrained short-term and working memory assessments (pretraining vs posttraining: $F_{(1,12)}=45.072, p<$ 0.001 ), but not so for the Placebo group (pretraining vs posttraining: $\left.F_{(1,13)}=3.572, p=0.081\right)$, resulting in a significant interaction between group and time $\left(F_{(1,24)}=20.500, p<0.001\right.$; Figure 2$)$. This effect did not differ significantly across the verbal or spatial measures from the assessment battery (3-way interaction: $F_{(3,72)}=0.436, p=0.728$ ).

\section{Adaptive training enhances resting functional connectivity}

We conducted a whole-brain connectivity analysis based upon the independent set of seed networks. These networks were chosen a priori because they are routinely linked with attentional control and working memory. In the lower beta-band (13-20 $\mathrm{Hz}$ ), we identified a significant effect of the group by time interaction involving connectivity between the right-hemisphere frontoparietal network and left lateral occipital cortex (LOC) that survived our multiple-comparisons correction $\left(p_{\text {corrected }}=\right.$ 0.0496; Figure $3 A$ ); that is, the two groups differ after training, but not before. Figure $3 B$ shows the mean connectivity values in the left LOC area for each group before and after training.

However, this is not the most direct way of exploring the neural effects of cognitive training because it does not take account of the actual change in working memory across the training period. In reality, the benefits of training are variable across children. For this reason, we used a second GLM that captured the linear relationship between individual gains in working memory and changes in functional connectivity. We observed two significant effects, both being lower beta-band connections with a bilateral frontoparietal network that comprised bilateral superior parietal cortex and frontal eye-fields (Fig. 4A). The first significant effect was within the network itself, in the left superior parietal lobule $\left(p_{\text {corrected }}=0.0392\right)$; the second was in left inferior temporal (IT) cortex $\left(p_{\text {corrected }}=0.0190\right)$ close to area IT. The relationship in these areas, between change in connectivity and change in ability, can be seen in Figure $4, B$ and $C$. Again, these results are corrected for multiple comparisons across all voxels, seed networks, and frequencies. We used these scatter plots to check that the significant relationships between changes in connectivity and changes in working memory capacity were not unduly biased by individual cases. To test this formally, we calculated Cook's distance, which quantifies the influence of each data point on the overall strength of the relationship. Across the plots shown in Figure $4, B$ and $C$, the highest Cook's distance value for any data point is 0.13 . This falls well below the typically used threshold of 1 . The same pattern, of increasing connectivity with increasing working memory gains, is present in the connection identified by the first GLM, a scatterplot of which can be seen in Figure $3 C$. There were no significant negative effects associated with the cognitive training.

\section{Resting-state networks at $13-20 \mathrm{~Hz}$}

The GLM analyses identified two resting-state networks that were altered by working memory training, both in the $13-20 \mathrm{~Hz}$ range. We subsequently tested whether our results reflect trainingrelated neurophysiological changes to preexisting functional networks at this frequency band. To do this, we conducted an additional analysis on the pretraining data. We submitted our source-projected temporally concatenated down-sampled envelope data at $13-20 \mathrm{~Hz}$ to a temporal ICA. This ICA reduces the 8417 location-specific time courses to a set of 25 underlying components that have temporally distinct power fluctuations in the 13-20 Hz range. Importantly, the ICA is blind to the spatial locations of those original recordings. The resulting component time courses could then be correlated with the original location-specific envelope time courses to produce spatial maps of the underlying components. This is the same approach used by Brookes et al. (2011) to study resting-state networks using oscillatory data. Even though we did not provide the ICA with any spatial information, two of the resulting component maps were highly overlapping with the networks from the independent $\mathrm{fc}$-fMRI dataset implicated by our analysis. Figure 5 shows the two networks significantly altered by the training. Also shown in Figure 5 are the component maps produced by our temporal ICA. We used the FSL tool fslcc to test whether there was significant spatial overlap between the two independent datasets by calculating spatial cross-correlations. These could then be converted into $z$ statistics to provide a statistical test of the spatial overlap. These show that these ICA-derived 13-20 Hz maps are indeed significantly related to their fc-fMRI counterparts (right-hemisphere fronto-parietal: $r=0.27, z=26.1638, p<0.001$; bilateral fronto-parietal: $r=$ $0.38, z=36.8142, p<0.001)$. This demonstrated that there was activity in the 13-20 Hz range in our networks of interest before training. Furthermore, it showed that the endogenous ongoing $13-20 \mathrm{~Hz}$ activity is robust enough that it can be used to define the spatial extent of the networks. 
A Dorsal

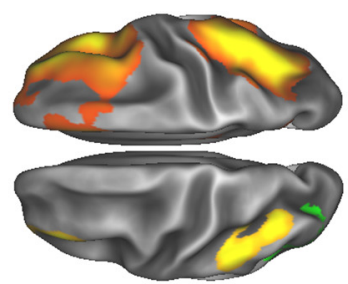

Left Hemisphere

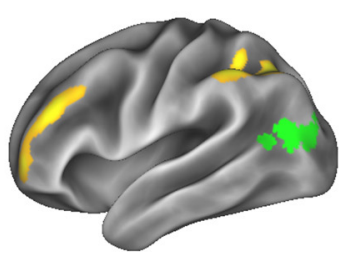

Right Hemisphere

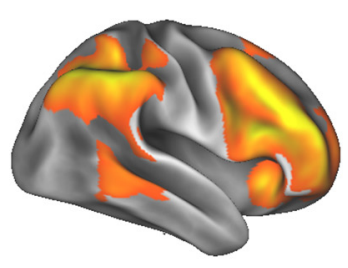

Ventral

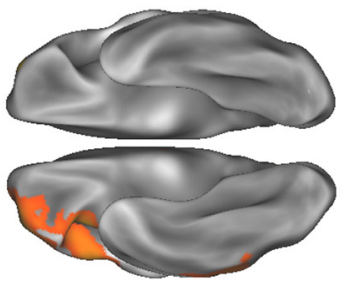

B区

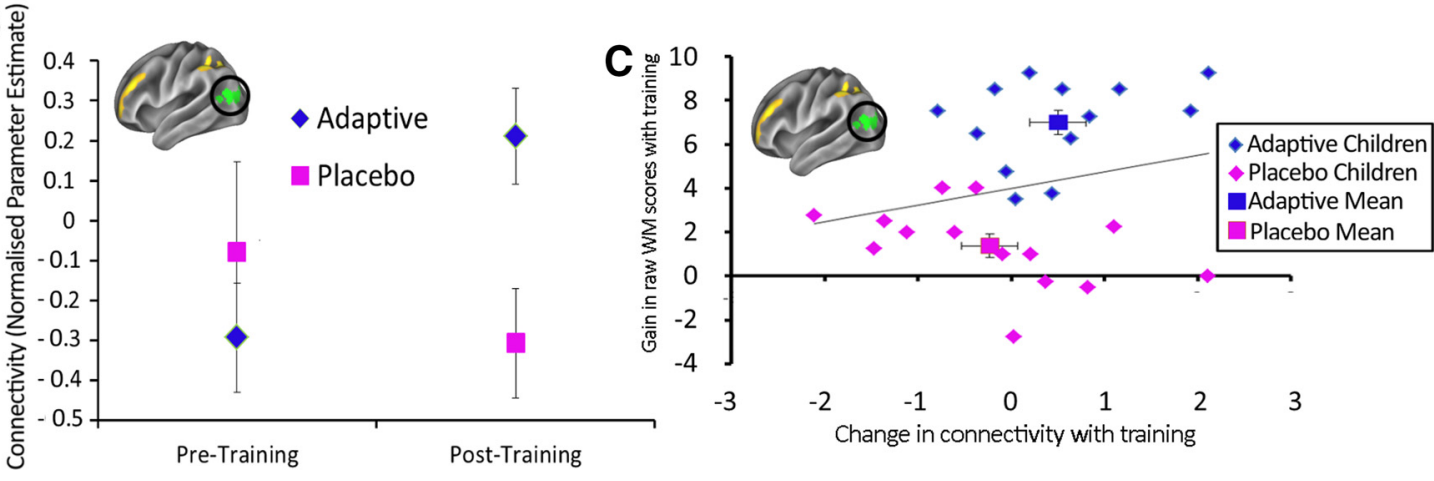

Figure 3. A, Red-orange areas correspond to a right hemisphere frontoparietal seed network (Smith et al., 2012). The green area highlights an area of functional interaction with this network that demonstrates a significant interaction between group (Adaptive vs Placebo) and time (pretraining versus posttraining) in the first GLM analysis, corrected for multiple comparisons across voxels, seed networks, and frequencies. $\boldsymbol{B}$, Connectivity values for left LOC for each intervention group before and after training. C, Changes in connectivity values for individual subjects across both intervention conditions plotted against changes in working memory capacity after training. In all cases, the error bars correspond to the SEM. All of the measures of connectivity show normalized parameter estimates from the GLM.

A
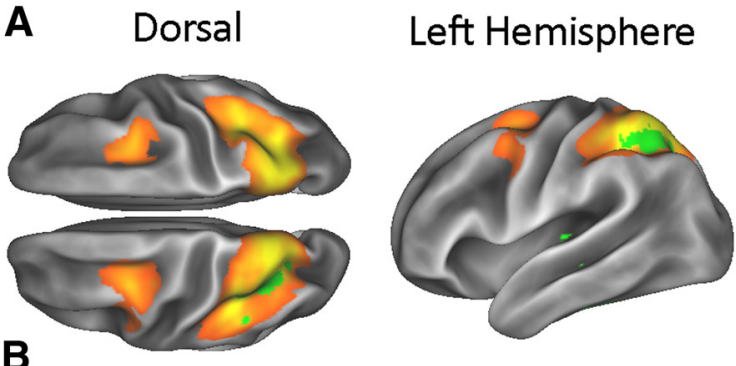

B

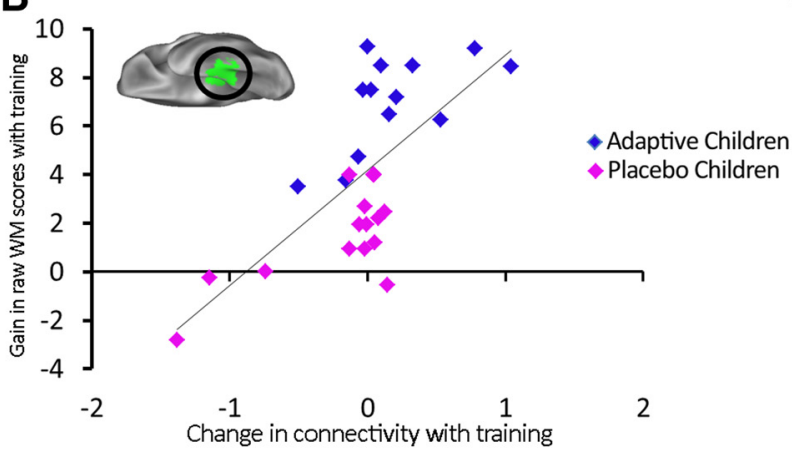

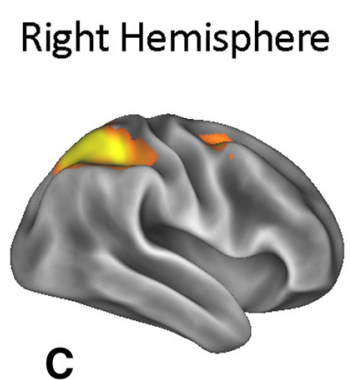
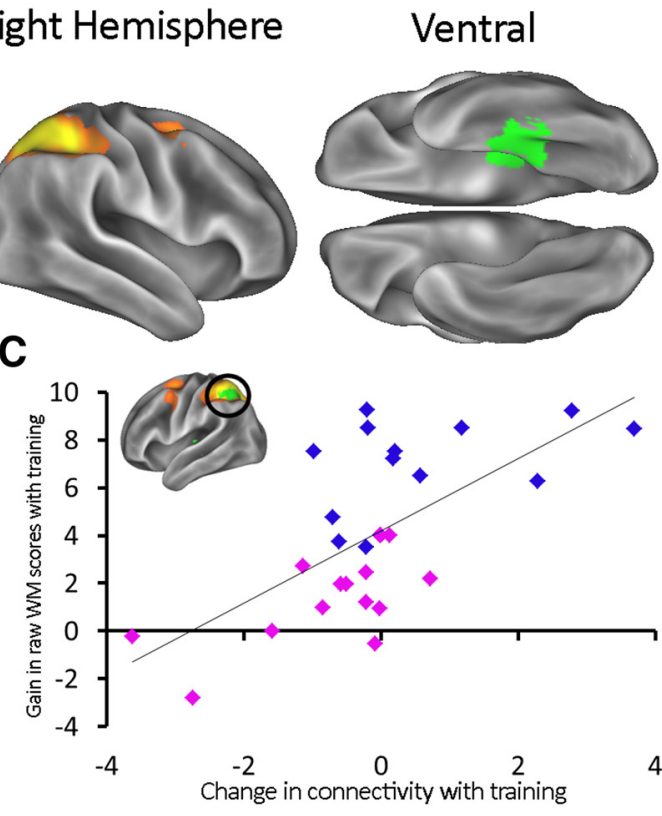

Figure 4. A, Red-orange areas correspond to a bilateral seed network including superior parietal and middle frontal gyri (Smith et al., 2012). The green area highlights significant effects of working memory ability posttraining while controlling for working memory ability pretraining. This result is corrected for multiple comparisons across voxels, seed networks, and frequencies. $\boldsymbol{B}$ and C, Relationship between changes in working memory ability (posttraining minus pretraining) and changes in connectivity in the inferior temporal cortex $(r=0.68, p<0.001)(\boldsymbol{B})$ and the superior parietal lobule $(r=0.65, p<0.001)$ (C). All of the measures of connectivity show normalized parameter estimates from the GLM.

\section{Discussion}

After 20 sessions of adaptive working memory training, children's working memory capacity was substantially increased, relative to a group of children that received a placebo intervention. These capacity gains were mirrored by changes in resting brain connectivity. We identified significant changes to connectivity between a right-lateralized frontoparietal network and left lateral occipital cortex with training. This result was apparent as a group-level training effect (Adaptive vs Placebo). We also observed considerable variation between individual subjects in the magnitude of change in working memory performance before and after the intervention. The second GLM analysis explored sources of this variation without presuming that the major determinant was adaptive versus placebo training condition. This identified a significant enhancement of connectivity between a bilateral frontoparietal network, superior parietal cortex, and a 


\section{Right-Hemisphere Fronto-Parietal Network}

\section{Left Hemisphere}
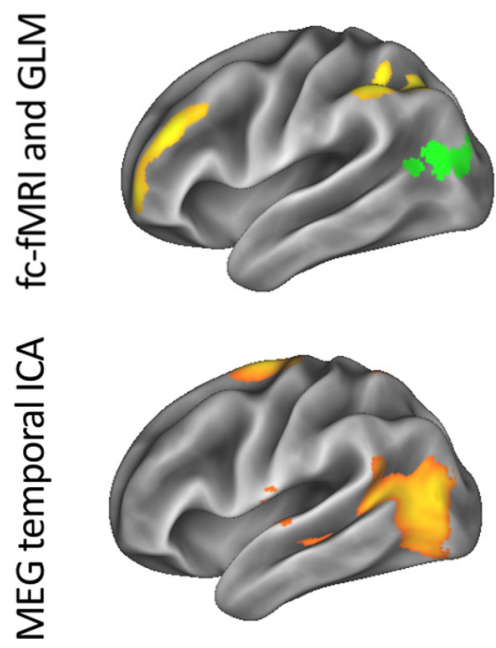

Right Hemisphere
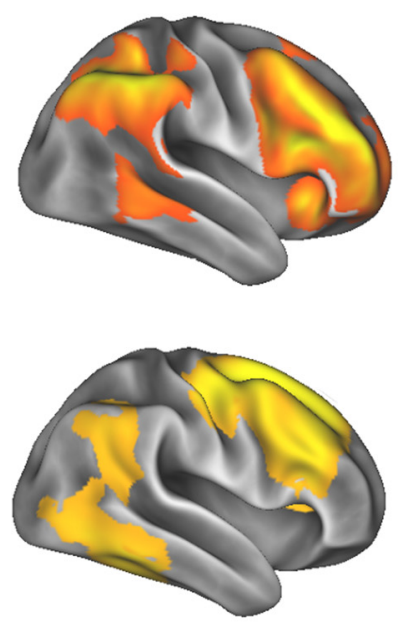

Bilateral Superior Fronto-Parietal

Left Hemisphere
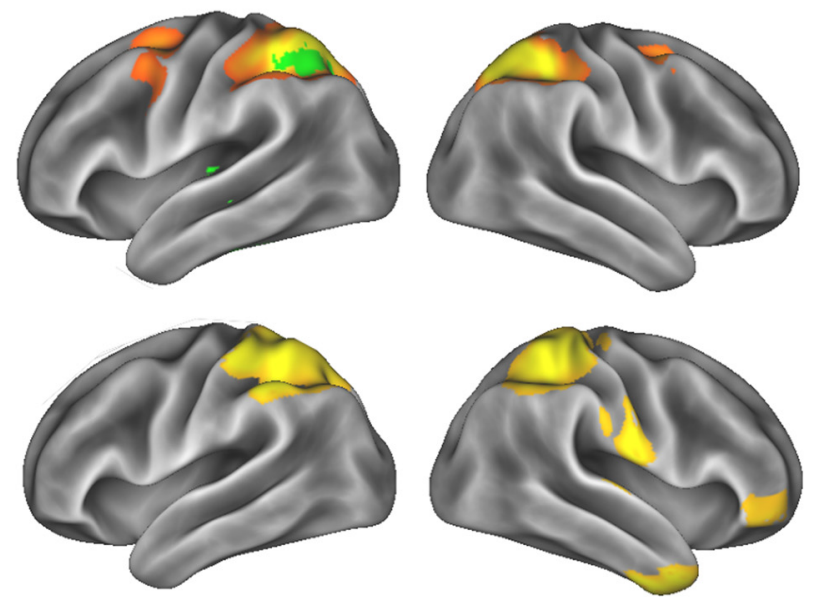

Figure 5. The two networks significantly altered after working memory training, which are spatially defined using an independent fc-fMRI dataset from Smith et al. (2012). As in previous figures, any green areas correspond to significant effects from either of the GLM analyses. The lower set of maps shows the result of a spatially blind temporal ICA conducted on the temporally down-sampled concatenated Hilbert envelope data, also in the $13-20 \mathrm{~Hz}$ range, before training.

portion of inferior temporal cortex close to area IT. Across these dynamic functional systems, connectivity changes associated with training were greatest in those who showed the biggest improvement in working memory capacity.

These associated changes in memory capacity and brain connectivity over time could be explained by differences between children in the rate of intrinsic neural and cognitive development occurring in parallel with the study. Although difficult to rule out with this type of analysis, it is important to remember that the time period of the intervention was relatively short and the age range of our sample is small. Even though all children will have developed over this period, which could have contributed to the overall difference between Time 1 and Time 2, this cannot explain the differential changes to capacity and connectivity that we see across the children. Furthermore, even though the second analysis did not include group as a factor, from the scatter plots, we can see that the strongest predictor of the size of working memory and connectivity change was group membership. Therefore, intrinsic developmental rate would have to be distributed unequally between groups by chance for this factor to explain the differences observed. We would suggest instead that the doubleblind, randomized-controlled design of the current study provides strong evidence that training-induced changes in working memory are associated with significant changes in resting brain connectivity.

Previous studies of the neurophysiological mechanisms of attentional control and working memory have highlighted the importance of long-range functional connections. For example, intracranial recording techniques have shown that neuronal oscillatory signals between the frontal eye fields (FEFs) and area V4 become synchronized when attentional control is engaged (Gregoriou et al., 2009). Similar processes have been observed in human subjects using BOLD-based imaging, with top-down inputs into LOC acting to bias processing in favor of relevant representations over competing representations (Stokes et al., 2009). These long-range connections, typically between areas in frontal and parietal cortex (including dorsolateral prefrontal cortex, the
FEFs, and the superior and inferior parietal cortex) and lowerlevel processing areas (including LOC and areas IT and V4), enable the dynamic regulation of ongoing processing according to fluctuating task goals (for review, see Desimone and Duncan, 1995). Our results demonstrate that working memory training is associated with the enhancement of these functional connections in childhood and that the magnitude of these specific neurophysiological changes is mirrored by individual gains in similarly structured but untrained working memory exercises. A plausible explanation for these results is that the enhanced coordination between these areas, apparent even at rest, allows for better regulation of ongoing sensory and cognitive processes and thereby affords greater performance on control demanding working memory exercises. However, this remains only a suggestion. With the current data alone, there remain a number of possibilities as to how adaptive training mediates the observed effects of neural processing and cognitive performance and why this effect varies between individuals, even between those subjected to the same training protocol. One possibility is that children vary in their degree of motivation and that this mediates the impact of the intervention. Furthermore, the increasing task difficulty in the adaptive condition may promote this motivation rather than, or in addition to, affecting specific cognitive processes.

Cognitive training was associated with altered connectivity within the 13-20 Hz frequency band and a subsequent analysis revealed that ongoing endogenous activity in this bandwidth can be used to characterize the networks implicated in the training effects; that is, the training influences ongoing rhythmic activity within these networks that is apparent before training. Top-down modulation of the alpha and lower beta activity within sensory cortex is consistently and critically linked to perceptual sensitivity (for review, see Hanslmayr et al., 2011). A popular mechanistic account of attentional control is that it is enacted via the modulation of relatively slow-frequency oscillations in sensory cortex in the alpha and lower beta range. The modulation of these rhythms represents a physiological mechanism by which task control centers can gate the ongoing processing of sensory input 
according to its relevance (van Ede et al., 2011). These same topdown modulatory signals are also present at different stages of working memory encoding, storage and retrieval (for review, see Gazzaley and Nobre, 2012). We suggest that working memory training may result in capacity gains because it intensively taxes these control mechanisms, resulting in their enhancement. This enhancement is apparent even at rest in the endogenous coordination of activity between areas in frontoparietal cortex and areas in inferior temporal cortex (close to area IT) and LOC. Moreover, these enhancements occur at a frequency previously associated with attentional control mechanisms (Hanslmayr et al., 2011).

This study represents the first systematic demonstration that cognitive training augments intrinsic neurophysiological brain connectivity in childhood. Because this effect is observed "at rest," this training-related difference in intrinsic connectivity cannot be attributed to differences in motivation or strategy per se (because the children are not performing any task during the scan). Furthermore, because we use MEG, which is a direct measure of neural activity, our effects cannot be attributed to differences in blood flow or metabolism (Schmithorst et al., 2015). A subsequent challenge is to test whether and how the identified mechanisms can be harnessed via cognitive training to produce therapeutic benefits that extend to aspects of everyday life for diverse target populations with either developmental or acquired deficits.

\section{References}

Alloway TP, Gathercole SE, Kirkwood H, Elliott J (2008) Evaluating the validity of the Automated Working Memory Assessment. Educational Psychology 28:725-734.

Anguera JA, Boccanfuso J, Rintoul JL, Al-Hashimi O, Faraji F, Janowich J, Kong E, Larraburo Y, Rolle C, Johnston E, Gazzaley A (2013) Video game training enhances cognitive control in older adults. Nature 501:97101. CrossRef Medline

Astle DE, Luckhoo H, Woolrich M, Kuo BC, Nobre AC, Scerif G (2014) The neural dynamics of fronto-parietal networks in childhood revealed using magnetoencephalography. Cereb Cortex. In press.

Barnes JJ, Woolrich MW, Baker K, Colclough GL, Astle DE (2015) Electrophysiological measures of resting state functional connectivity and their relationship with working memory capacity in childhood. Dev Sci. Advance online publication. doi:10.1111/desc.12297. CrossRef Medline

Brookes MJ, Woolrich M, Luckhoo H, Price D, Hale JR, Stephenson MC, Barnes GR, Smith SM, Morris PG (2011) Investigating the electrophysiological basis of resting state networks using magnetoencephalography. Proc Natl Acad Sci U S A 108:16783-16788. CrossRef Medline

Desimone R, Duncan J (1995) Neural mechanisms of selective visual attention. Annu Rev Neurosci 18:193-222. CrossRef Medline

Duncan J (2010) The multiple-demand (MD) system of the primate brain: mental programs for intelligent behaviour. Trends Cogn Sci 14:172-179. CrossRef Medline

Dunning DL, Holmes J, Gathercole SE (2013) Does working memory training lead to generalized improvements in children with low working memory? A randomized controlled trial. Dev Sci 16:915-925. Medline

Filippini N, MacIntosh BJ, Hough MG, Goodwin GM, Frisoni GB, Smith SM, Matthews PM, Beckmann CF, Mackay CE (2009) Distinct patterns of brain activity in young carriers of the APOE-epsilon4 allele. Proc Natl Acad Sci U S A 106:7209-7214. CrossRef Medline

Gathercole SE, Pickering SJ, Knight C, Stegmann Z (2003) Working memory skills and educational attainment: evidence from national curriculum assessments at 7 and 14 years of age. Applied Cognitive Psychology 18:1-16.

Gazzaley A, Nobre AC (2012) Top-down modulation: bridging selective attention and working memory. Trends Cogn Sci 16:129-135. CrossRef Medline

Gregoriou GG, Gotts SJ, Zhou H, Desimone R (2009) High-frequency, long-range coupling between prefrontal and visual cortex during attention. Science 324:1207-1210. CrossRef Medline

Hanslmayr S, Gross J, Klimesch W, Shapiro KL (2011) The role of alpha oscillations in temporal attention. Brain Res Rev 67:331-343. CrossRef Medline

Holmes J, Gathercole SE, Dunning DL (2009) Adaptive training leads to sustained enhancement of poor working memory in children. Dev Sci 12:F9-F15. CrossRef Medline

Jolles DD, van Buchem MA, Rombouts SA, Crone EA (2012) Practice effects in the developing brain: a pilot study. Dev Cogn Neurosci 2:S180-S191. CrossRef Medline

Kilner JM (2013) Bias in a common EEG and MEG statistical analysis and how to avoid it. Clin Neurophysiol 124, 2062-2063. CrossRef Medline

Klingberg T, Fernell E, Olesen PJ, Johnson M, Gustafsson P, Dahlström K, Gillberg CG, Forssberg H, Westerberg H (2005) Computerized training of working memory in children with ADHD-a randomized, controlled trial. J Am Acad Child Adolesc Psychiatry 44:177-186. CrossRef Medline

Luckhoo H, Hale JR, Stokes MG, Nobre AC, Morris PG, Brookes MJ, Woolrich MW (2012) Inferring task-related networks using independent component analysis in magnetoencephalography. Neuroimage 62:530541. CrossRef Medline

Luckhoo HT, Brookes MJ, Woolrich MW (2014) Multi-session statistics on beamformed MEG data. Neuroimage 95:330-335. CrossRef Medline

Rueda MR, Checa P, Cómbita LM (2012) Enhanced efficiency of the executive attention network after training in preschool children: immediate changes and effects after two months. Dev Cogn Neurosci 2:S192-S204. CrossRef Medline

Schmithorst VJ, Vannest J, Lee G, Hernandez-Garcia L, Plante E, Rajagopal A, Holland SK; CMIND Authorship Consortium (2014) Evidence that neurovascular coupling underlying the BOLD effect increases with age during childhood. Hum Brain Mapp 36:1-15. Medline

Schoffelen JM, Gross J (2009) Source connectivity analysis with MEG and EEG. Hum Brain Mapp 30:1857-1865. CrossRef Medline

Smith SM, Miller KL, Moeller S, Xu J, Auerbach EJ, Woolrich MW, Beckmann CF, Jenkinson M, Andersson J, Glasser MF, Van Essen DC, Feinberg DA, Yacoub ES, Ugurbil K (2012) Temporally-independent functional modes of spontaneous brain activity. Proc Natl Acad Sci U S A 109:3131-3136. CrossRef Medline

Stokes M, Thompson R, Nobre AC, Duncan J (2009) Shape-specific preparatory activity mediates attention to targets in human visual cortex. Proc Natl Acad Sci U S A 106:19569-19574. CrossRef Medline

Stokes MG (2011) Top-down visual activity underlying VSTM and preparatory attention. Neuropsychologia 49:1425-1427. CrossRef Medline

van Ede F, de Lange F, Jensen O, Maris E (2011) Orienting attention to an upcoming tactile event involves a spatially and temporally specific modulation of sensorimotor alpha-and beta-band oscillations. J Neurosci 31: 2016-2024. CrossRef Medline

Van Veen BD, van Drongelen W, Yuchtman M, Suzuki A (1997) Localization of brain electrical activity via linearly constrained minimum variance spatial filtering. IEEE Trans Biomed Eng 44:867-880. CrossRef Medline

Westerberg H, Jacobaeus H, Hirvikoski T, Clevberger P, Ostensson ML, Bartfai A, Klingberg T (2007) Computerized working memory training after stroke-a pilot study. Brain Inj 21:21-29. CrossRef Medline

Woolrich M, Hunt L, Groves A, Barnes G (2011) MEG beamforming using Bayesian PCA for adaptive data covariance matrix regularization. Neuroimage 57:1466-1479. CrossRef Medline 